\title{
CALIDAD AMBIENTAL DE LOS RÍOS CURARAY, ARABELA Y NAPO (LORETO, PERÚ)
}

\author{
Werner CHOTA-MACUYAMA ${ }^{1 *}$, Fred CHU KOO ${ }^{1}$, Carmen GARCÍA-DÁVILA ${ }^{1}$, Diana CASTRO-RUIZ1, \\ Rosa ISMIÑO ${ }^{1}$, Aurea GARCÍA ${ }^{1}$, Homero SÁNCHEZ ${ }^{1}$, Lamberto ARÉVALO ${ }^{1}$, Salvador TELLO ${ }^{1}$ \\ 1 Instituto de Investigaciones de la Amazonía Peruana - IIAP. Programa para el Uso y Conservación del Agua y sus Recursos \\ -AQUAREC. Apartado 784. Iquitos. Perú. *e-mail:wchota@iiap.org.pe
}

\section{RESUMEN}

Se evaluó la calidad ambiental de los ríos Curaray, Arabela y Napo (a la altura de la desembocadura del Curaray) a través de la determinación de sus parámetros físicos y químicos, la presencia de aceites y grasas, hidrocarburos totales de petróleo y metales totales en agua, sedimentos y músculo de peces, durante los períodos de creciente y vaciante del año 2012. Los resultados del análisis de agua en siete estaciones de muestreo, en ambos períodos hidrológicos, mostraron que el agua de los ríos, a excepción de la Estación Curaray 1 (Arica), estuvieron libres de aceites y grasas, hidrocarburos totales de petróleo y de metales pesados como el mercurio, cadmio, plomo y arsénico. En el período de creciente, las concentraciones de mercurio, cadmio, cromo, plomo, níquel y zinc en los sedimentos estuvieron por debajo de los estándares internacionales tomados como referencia, a excepción del cobre en la Estación Napo 1 (Nuevo Defensor). En la vaciante, solo se registraron concentraciones de mercurio por encima de los niveles permisibles $(0,27$ a $0,30 \mathrm{mg} / \mathrm{kg}$ ) en los sedimentos de la Estación Curaray 3 (Shapajal). Finalmente, las concentraciones de mercurio detectadas en los peces colectados $(0,03-0,07 \mathrm{mg} / \mathrm{kg})$ estuvieron por debajo de los niveles permisibles.

PALABRAS CLAVE: Características limnológicas, hidrocarburos, metales, sedimentos, peces.

\section{ENVIRONMENTAL QUALITY OF THE CURARAY, ARABELA AND NAPO RIVERS (LORETO, PERÚ)}

\section{ABSTRACT}

The aim of this study was to evaluate the environmental quality of the Arabela, Curaray and Napo Rivers through the determination of their physicochemical parameters, the presence of oil, grease, total petroleum hydrocarbons and total metals in water, sediments and fish tissues during the growing and dry season of 2012. The results of the water analysis in seven sampling stations in both hydrological periods, except for Curaray 1 station (Arica), were free of oil and grease, total petroleum hydrocarbons and heavy metals such as mercury, cadmium, lead and arsenic. In the river's wet season, sediments showed concentrations of heavy metals (mercury, cadmium, chromium, lead, nickel and zinc) within the permissible international standards taken as reference; except for copper in Napo 1 station (Nuevo Defensor). In the dry season, concentrations of mercury above the permissible levels $(0.27-0.30 \mathrm{mg} / \mathrm{kg})$ were only recorded in sediments sampled from Curaray 3 station (Shapajal). Finally, concentrations of mercury detected in fish $(0.03-0.04 \mathrm{mg} / \mathrm{kg})$ were below the maximum permissible limit. With our results it was concluded that, the physical and chemical quality of the rivers studied in relation to the parameters evaluated during 2012, were in appropriate conditions for the development of aquatic life.

KEYWORDS: limnological characteristics, hidrocarbons, metals, sediments, fishes. 


\section{INTRODUCCIÓN}

La Amazonía cuenta con una amplia oferta de recursos hídricos, que excede la demanda de uso de los mismos (Programa de las Naciones Unidas et al., 2009), sin embargo, el deterioro de su calidad es uno de los problemas ambientales más importantes. Como ejemplo de ello, en el año 2013, el Ministerio del Ambiente declaró en emergencia ambiental a los ríos Pastaza, Corrientes y Tigre a consecuencia de la actividad petrolera. Asimismo, el año 2012, la Autoridad Nacional del Agua (ANA) registró en muestras de agua de la cuenca del Nanay y sus afluentes, concentraciones de mercurio y plomo por encima de los niveles permitidos (ANA, 2012). La ANA considera que la calidad del agua del Nanay está influenciada por los botaderos de residuos domésticos, las actividades de dragado y las aguas residuales domésticas, pudiendo también provenir, en el caso de los metales, de la mineralogía de la cuenca.

La vulnerabilidad de los recursos hídricos radica principalmente en su alto poder para disolver y transportar gran variedad de sustancias de desecho (Pabon \& Chaparro, 2010). Por tanto, sus características pueden alterarse con sustancias como los aceites, grasas, hidrocarburos y metales que provienen de fuentes antrópicas tales como la deforestación, minería, extracción de hidrocarburos, agricultura, transporte, vertidos industriales, aguas domésticas, entre otras (Vitousek, 1994; Simeonov et al., 2003; Programa de las Naciones Unidas et al., 2009, Christophoridis et al., 2009), suponiendo una amenaza para el medio ambiente y la salud pública. Los casos más conocidos son los relacionados con metales pesados, que tienden a bioacumularse y biomagnificarse en los organismos que componen la cadena alimenticia, pudiendo afectar seriamente al ser humano (Diagomanolin et al., 2004).

En este contexto, y ante el incremento de actividades antrópicas, es importante conocer el estado actual de las cuencas amazónicas. Por lo que el objetivo del estudio fue evaluar la calidad ambiental de los ríos Curaray, Arabela y Napo (a la altura de la desembocadura del Curaray) a través de la determinación de sus parámetros físicos y químicos, la presencia de aceites y grasas, hidrocarburos totales de petróleo y metales totales en agua, sedimentos y músculo de peces, durante los períodos de creciente y vaciante del año 2012.

\section{MATERIALES Y MÉTODOS}

\section{Área de estudio y estaciones de muestreo}

El estudio se desarrolló en los ríos Curaray, Arabela y Napo (a la altura de la desembocadura del
Curaray), ubicados en el distrito del Napo, provincia de Maynas, región Loreto. Las muestras de agua, sedimentos y peces fueron colectadas de siete estaciones de muestreo (Figura 1), durante los períodos hidrológicos de creciente (entre abril y mayo) y vaciante (entre octubre y noviembre) del año 2012.

\section{Colecta de muestras}

Agua: Para los análisis de aceites y grasas, hidrocarburos totales de petróleo (HTP) y metales fueron colectadas 14 muestras de agua, dos por cada estación de muestreo (una de la orilla y otra del centro). Las muestras para aceites y grasas fueron preservadas con 40 gotas de ácido sulfúrico (diluido 1:1), para metales con 20 gotas de ácido nítrico (diluido 1:1) y para HTP no se utilizaron preservantes. Todas las muestras fueron mantenidas en refrigeración como máximo 15 días hasta su análisis.

Sedimentos: Utilizando una draga tipo Eckman, se colectaron dos muestras de sedimento por cada estación de muestreo (una de la orilla y otra del centro del río). Las muestras fueron depositadas en frascos de vidrio color ámbar de $1 \mathrm{~L}$ y conservadas en refrigeración, sin preservantes.

Peces: Se colectó un espécimen por cada estación de colecta, tratando de cubrir en conjunto todas las categorías tróficas. La pesca, en la época de creciente, fue realizada en zonas inundables o en la entrada de los caños que hacen la conexión con los lagos, empleando redes agalleras de 7,5 $\mathrm{cm}$ y 10,0 $\mathrm{cm}$ de abertura de malla. En la vaciante se emplearon además, anzuelos y tarrafas. Los ejemplares capturados fueron medidos y pesados, luego se tomó entre 150 a $250 \mathrm{~g}$ de tejido muscular (dependiendo del tamaño). Las muestras fueron conservadas en un congelador sin preservantes.

\section{Análisis de las muestras e interpretación de los resultados}

Los análisis de aceites y grasas, HTP y metales totales fueron realizados por la empresa acreditada Environmental Laboratories Perú S.A.C. Los metales evaluados fueron el aluminio, antimonio, arsénico, bario, berilio, bismuto, boro, cadmio, calcio, cobalto, cobre, cromo, estaño, estroncio, fósforo, hierro, litio, magnesio, manganeso, mercurio, molibdeno, níquel, plata, plomo, potasio, selenio, silicio, sodio, titanio, talio, vanadio y zinc.

Análisis de agua: En cada estación de muestreo se tomaron datos limnológicos in situ. Para ello se utilizó un equipo multiparámetros (YSI, modelo MPS 556), que registró los siguientes parámetros: 
temperatura del agua, oxígeno disuelto, conductividad eléctrica, $\mathrm{pH}$ y sólidos totales disueltos (STD). Las concentraciones de amonio, nitritos, dióxido de carbono $\left(\mathrm{CO}_{2}\right)$, cloruros, dureza total, la alcalinidad total fueron determinadas por un equipo para análisis de aguas dulces (LaMotte, Kit AQ-2) y la transparencia por un disco Secchi. Los aceites y grasas fueron analizados mediante el método EPA 1664-A, los HTP por el EPA 8015-C, la corrida de metales por el EPA 200.7, y el mercurio por el EPA METHOD 245.7.

Análisis de sedimentos: Los aceites y grasas fueron analizados mediante el método SM 5520-E, los HTP (C10-C40) por el EPA 8015-C, la corrida de metales (Óptico) por el EPA 6010-B, y el mercurio por el Método (ICP-AES) EPA 7474.

Análisis de peces: Para el análisis de aceites y grasas se utilizó el método SM 5520, para HTP fue el EPA 8015-C, para la corrida de metales fue el EPA 6010-B y para el mercurio fue el Método CVAFS.

Valores estándares utilizados. Para la interpretación de los resultados, los datos obtenidos fueron comparados con valores estándares de calidad ambiental. En la mayoría de los casos nuestros datos fueron comparados con valores estándares de otros países, debido a que en el Perú todavía no han sido establecidos. En el caso del agua, los estándares utilizados fueron: A) Estándares de Calidad Ambiental para Aguas de Categoría 4: Conservación del Ambiente Acuático, del Ministerio del Ambiente (MINAM) de Perú, 2008; B) Directiva de Consejo 78/659/CEE de la Unión Europea (UE); C) Criterios de calidad admisibles para la preservación de la flora y fauna en aguas dulces, frías o cálidas, y en aguas marinas y de estuario de Colombia; y D) Estándares de Calidad Ambiental para Aguas de Categoría 1: Poblacional y Recreacional. A1: Aguas que pueden ser potabilizadas con desinfección, del Ministerio del Ambiente (MINAM) de Perú. Para sedimentos se utilizaron estándares ambientales de los siguientes países: F) Estados Unidos (EPA Region IV. Ecological Screening Values - ESV) y G) Holanda (Dutch Standards. Intervention values and Target values - Soil quality standards). Para peces se utilizaron los valores estándares establecidos por la I) Divisão Nacional de Vigilância Sanitária de Alimentos - DINAL (Brasil), J) Organización Mundial de la Salud (OMS), y L) Conselho Nacional de Meio Ambiente - CONAMA, Resolução 3572005 (Brasil).

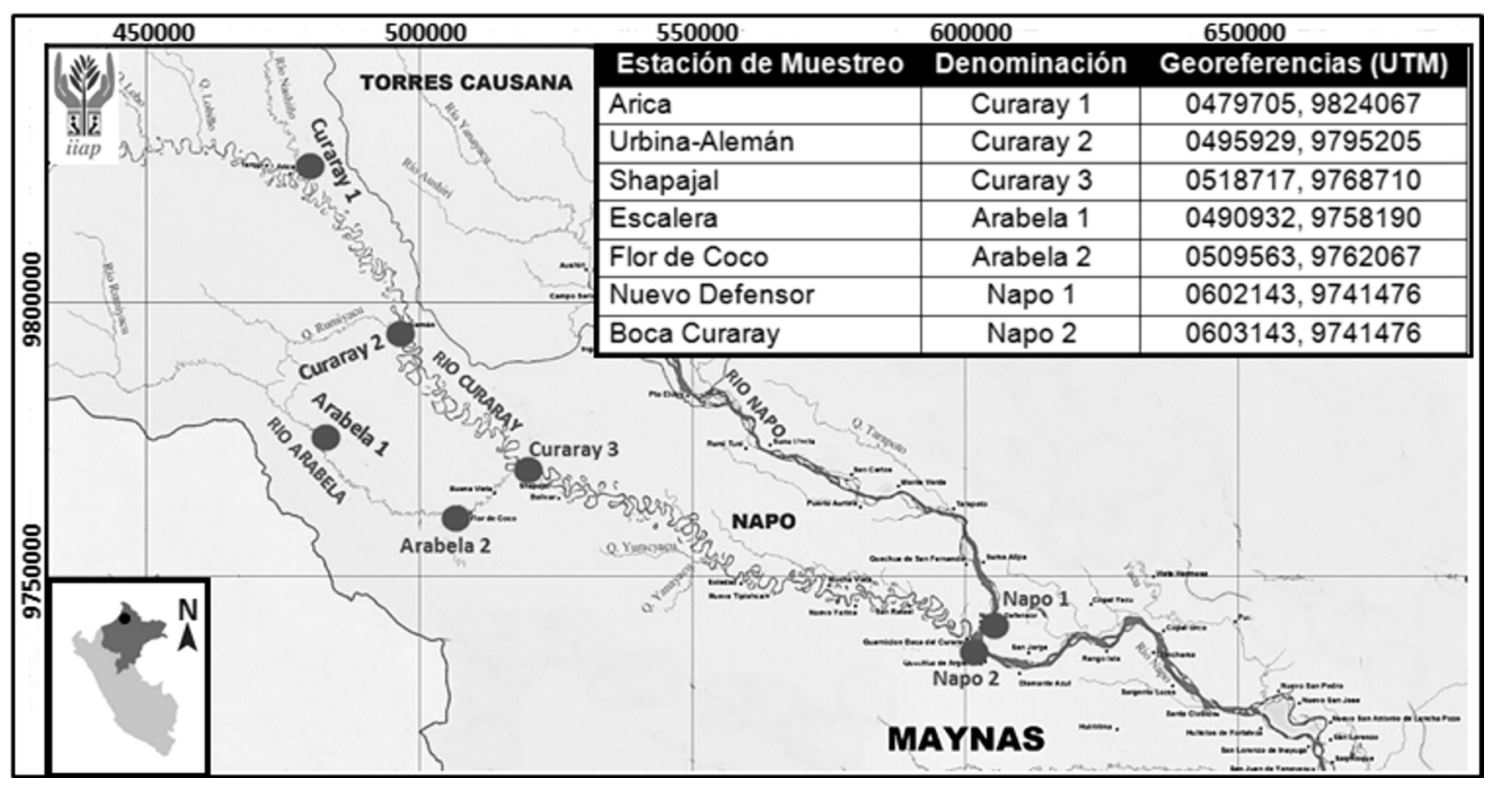

Figura 1. Mapa de ubicación, denominación y georeferencia de las siete estaciones de colecta en los ríos Curaray, Arabela y Napo. 


\section{RESULTADOS Y DISCUSIÓN}

\section{Características limnológicas}

Los ríos amazónicos pueden clasificarse según las características físicas y químicas del agua en "ríos de aguas blancas", "ríos de aguas negras", "ríos de aguas claras" y "ríos de aguas intermedias". Todos ellos, dependiendo de su origen y las zonas que drenan, presentan diferencias en conductividad, sólidos totales disueltos, transparencia y $\mathrm{pH}$ principalmente (Sioli, 1984; Maco, 2006). Los datos obtenidos nos permitieron diferenciar ríos de aguas blancas, negras e intermedias.

El río Curaray presentó características típicas de aguas intermedias (Tabla 1). Sus aguas fueron blandas, con poco contenido de sólidos totales disueltos $(5,5-18 \mathrm{mg} / \mathrm{L})$ y baja conductividad eléctrica $(6,5-28 \mu \mathrm{S} / \mathrm{cm})$. El pH fluctuó entre 6,2-7,5 y la transparencia entre $12-30 \mathrm{~cm}$. Presentó moderada presencia de $\mathrm{CO}_{2}(3-5 \mathrm{mg} / \mathrm{L})$ y temperaturas entre $24,4-27,5^{\circ} \mathrm{C}$. Mientras que el río Arabela (afluente del Curaray) presentó características de aguas negras, debido al bajo contenido de sólidos totales disueltos $(6-14 \mathrm{mg} / \mathrm{L})$, baja conductividad $(9-21 \mu \mathrm{S} / \mathrm{cm})$, marcada acidez $(\mathrm{pH} 5,7-7,0)$ y mayor transparencia que la del Curaray $(20-50 \mathrm{~cm})$. El $\mathrm{CO}_{2}$ fue moderado, de 2-7 $\mathrm{mg} / \mathrm{L}$ y la temperatura varió de $24,9-30,2{ }^{\circ} \mathrm{C}$. Las características físicas y químicas del río Napo (a la altura de la desembocadura del Curaray) fueron muy diferentes a la de sus tributarios (Curaray y Arabela), presentando características típicas de los ríos de aguas blancas. El contenido de sólidos totales disueltos (15-37 mg/L) y la conductividad (23-56 $\mu \mathrm{S} / \mathrm{cm})$ fueron mayores. El $\mathrm{pH}$ fue alcalino $(7,1-7,6)$ y la transparencia fue de $17-30 \mathrm{~cm}$. Además, el Napo presentó mayores valores de dureza $(8-20 \mathrm{ppm}$ de $\mathrm{CaCO}_{3}$ ) y alcalinidad $(12-24 \mathrm{mg} / \mathrm{L})$. La temperatura de sus aguas osciló entre 25,7-28,7 ${ }^{\circ} \mathrm{C}$. Las diferencias en las características físicas y químicas de los ríos estudiados, según su origen, radica en que el Curaray y Arabela drenan zonas planas (valores bajos), mientras que el Napo (valores altos) provienen de los Andes (Granado, 2000; Ferreira et al., 2007). Finalmente, los niveles de oxígeno de los tres ríos fueron siempre cercanos o superiores a 5 $\mathrm{mg} / \mathrm{L}$, lo que permite cubrir ampliamente todas las actividades metabólicas de los organismos acuáticos que los habitan.

\section{Aceites y grasas, HTP y metales en aguas}

Los contaminantes metálicos son el mayor problema para la calidad de las aguas continentales, siendo uno de los factores principales que afectan a la salud de los ecosistemas como a la poblacional. En contraste a los contaminantes orgánicos, como los aceites, grasas e hidrocarburos, los metales no se degradan o eliminan; sus formas físicas y químicas cambian y son fácilmente removidos en el medio ambiente por mecanismos de transformación natural (Seyler \& Boaventura, 2003). La determinación de estos elementos en ambientes acuáticos es importante para evaluar la capacidad de recepción de las aguas para acumular contaminantes sin efectos perjudiciales. En nuestro estudio, en los períodos de creciente y vaciante, no se registró presencia de aceites y grasas o HTP en las estaciones de muestreo.

No fueron detectados metales como el cadmio, plomo, y mercurio, plata, boro, berilio, bismuto, cobalto, antimonio, selenio, estaño, talio, molibdeno, níquel, cromo y arsénico en la época de creciente de los ríos Curaray, Arabela y Napo (Tabla 2 ),. En la vaciante, se pudo comprobar que la presencia de metales varió con respecto a la creciente (Tabla 2). Se determinó la presencia de cobalto (Curaray y Napo), cromo y antimonio (Curaray), que no fueron detectados en creciente. Sin embargo, no se registraron (no fueron incluidos en las tablas por este motivo) metales como el boro, berilio, bismuto, cadmio, plomo, selenio, talio, plata, mercurio y arsénico. Otros metales detectados, tanto en creciente como en vaciante, fueron el litio, molibdeno, silicio, estaño, estroncio y titanio (pero no fueron incluidos en las tablas por carecer de valores estándares para su comparación). Otros como el bario, cobalto, cromo, níquel, vanadio y zinc, fueron encontrados por debajo de los Estándares Nacionales de Calidad Ambiental (ECA) para el Agua, establecidos en la norma nacional vigente (D.S. N $^{\circ}$ 002-2008-MINAM) para las Aguas de Categoría 4 (Conservación del Ambiente Acuático) y Categoría 1-A1 (Uso Poblacional y Recreacional-Aguas que pueden ser potabilizadas con desinfección).

En el río Curaray, tanto en creciente como en vaciante, las concentraciones de los metales por encima de los ECA para agua (Categoría 1, uso poblacional y recreacional) recomendados por el MINAM (2008), fueron el aluminio (0,49-1,97 $\mathrm{mg} / \mathrm{L}$, en las tres estaciones de colecta) y el hierro (1,36-3,52 $\mathrm{mg} / \mathrm{L}$, en las tres estaciones de colecta); en tanto que el manganeso, en creciente, sólo en la estación Curaray $1(0,13 \mathrm{mg} / \mathrm{L})$ y en vaciante en las estaciones Curaray $1(0,15-0,16 \mathrm{mg} / \mathrm{L})$ y Curaray 2 $(0,15 \mathrm{mg} / \mathrm{L})$. Los mayores valores fueron registrados en vaciante (Tabla 2). En vaciante se registró antimonio sólo en la estación Curaray 3 (orilla). En el río Arabela, tanto en creciente como en vaciante, los metales por encima de los valores permitidos fueron similares a los del Curaray: aluminio $(0,15-$ 
$0,39 \mathrm{mg} / \mathrm{L})$ y hierro $(0,9-1,7 \mathrm{mg} / \mathrm{L})$, en las dos estaciones de colecta. Los valores mayores para el aluminio fueron registrados en creciente, mientras que para el hierro fueron en vaciante. En el río Napo, para ambos períodos hidrológicos, los metales por encima de los ECA para agua (Categoría 4, conservación del ambiente acuático) fueron el aluminio $(0,32-1,66 \mathrm{mg} / \mathrm{L})$ y el hierro $(0,66-2,17$ $\mathrm{mg} / \mathrm{L}$ ), en las dos estaciones de colecta. Además de forma similar, en la creciente fue registrado el cobre $(0,051 \mathrm{mg} / \mathrm{L})$ y en la vaciante el fósforo $(0,12-0,16$ $\mathrm{mg} / \mathrm{L}$ ), ambos en las dos estaciones de colecta. Mientras que, tanto en creciente como en vaciante, el manganeso $(0,10-0,13 \mathrm{mg} / \mathrm{L})$ y el fósforo $(0,12-0,16$ $\mathrm{mg} / \mathrm{L}$ ) estuvieron cerca y sobre el límite superior de los valores permitidos ( $0,1 \mathrm{mg} / \mathrm{L}$ para ambos).

En nuestro estudio se pudo comprobar que el aluminio y el hierro fueron los metales más abundantes, con valores por encima de los ECA para agua en los tres ríos evaluados. Esto concuerda con el hecho de que los suelos de la cuenca amazónica pertenecen en su mayoría a la familia del suelo Ferralítico Rojo (que representan el $80 \%$ de la cuenca amazónica), cuya mineralogía es dominada por el cuarzo, óxidos de aluminio, fierro y caolinita, principalmente. Es decir, que la concentración registrada de estos elementos, al igual que el manganeso (que pertenece al mismo tipo de suelo), sugieren que son liberados de los suelos durante las altas descargas (Seyler \& Boaventura, 2003). En el caso particular del aluminio, se ha demostrado que este elemento es tóxico para los peces en el hemisferio norte. Pero poco se conoce de sus efectos en peces neotropicales, siendo uno de los escasos ejemplos el Prochilodus lineatus, con el cual se ha demostrado en ensayos de laboratorio que la concentración superior del ECA para agua de Brasil $(0.2 \mathrm{mg} / \mathrm{L}$, utilizado para comparación en el estudio) produce serias alteraciones en la osmorregulación del pez (Camargo et al. 2009). Similarmente, el cobre (registrado sólo en el Napo, en creciente) es un elemento ampliamente distribuido en los ecosistemas acuáticos, debido a que es un elemento de origen natural, pero sin embargo, las concentraciones pueden ser en gran medida incrementadas por fuentes antropogénicas, como derrames de petróleo (Adewuyi \& Olowu, 2012) o la quema de combustibles.

Tanto en creciente y vaciante, las concentraciones de los metales conocidos como macronutrientes (sodio, potasio, calcio y magnesio) fueron similares, siendo el más abundante el calcio, seguido del sodio, magnesio y potasio. Además, las concentraciones de estos metales fueron mayores en el Napo, seguido del Curaray y Arabela. La concentración de fósforo fue también uno de los indicadores de nuestros resultados, debido a que este metal está relacionado al contenido de sólidos disueltos, por tanto, mayor cantidad de fósforo indica mayor cantidad de sólidos (Ferreira et al., 2007). Si bien es cierto que las concentraciones fueron bajas, los sólidos disueltos fueron mayores en el Napo, seguido por el Curaray y el Arabela.

Nuestros datos difieren del estudio realizado por el GOREL-DIGESA (2012) tanto en número de metales detectados y en la concentración de los mismos, pudiendo ser debido a que los lugares de muestreo fueron diferentes. Pero todos las concentraciones al igual que en nuestro estudio estuvieron por debajo de los ECA para agua recomendado por el MINAM (2008). El estudio registró entre los metales más importantes al arsénico, cadmio, plomo y cromo. Como ejemplo, podemos mencionar al arsénico cuya concentración en el Curaray fue de $0,0006 \mathrm{mg} / \mathrm{L}$ (cerca de la comunidad de San Rafael), registrándose las mayores concentraciones en el río Napo, en la línea de frontera con el Ecuador $(0,0017 \mathrm{mg} / \mathrm{L})$ y cerca de la comunidad de Rango Isla $(0,0016 \mathrm{mg} / \mathrm{L})$.

\section{Aceites y grasas, HTP y metales en sedimentos}

En el período de creciente no se registró la presencia de aceites, grasas o HTP. Mientras que en la vaciante se encontraron aceites, grasas $(81 \mathrm{mg} / \mathrm{kg})$ y HTP $(191 \mathrm{mg} / \mathrm{kg})$ sólo en la estación Curaray 1 (Arica). En relación a los metales, en la creciente se determinó la presencia de 19 metales de los 32 analizados (Tabla 3). En este período no fueron detectados el boro, berilio, bismuto, cadmio, litio, molibdeno, antimonio, selenio, estaño, talio, plata, mercurio y arsénico. En la vaciante se registró el mayor número de metales (26), algunos de ellos en concentraciones más elevadas que en la creciente y por encima de los niveles permisibles (Tabla 3 ). En este período no fueron registrados el berilio, bismuto, cadmio, antimonio, talio y plata. En el período de creciente, se registró cobre sólo en una estación de muestreo (Napo 1) presentando concentraciones de $39,3 \mathrm{mg} / \mathrm{kg}$, superiores 2,1 veces al nivel máximo permitido por la agencia gubernamental estadounidense EPA región IV (18.7 $\mathrm{mg} / \mathrm{kg}$ ). Del mismo modo, en la misma estación, el níquel $(17 \mathrm{mg} / \mathrm{kg})$ fue registrado cerca al límite superior $(18 \mathrm{mg} / \mathrm{kg})$ de los mismos estándares.

El metal más importante por su toxicidad registrado en vaciante fue el mercurio, responsable de intoxicaciones y contaminación del medio ambiente (Driscoll et al., 2013; Sampaio et al., 2006). El mercurio se presenta como un importante riesgo para la salud de la población, ya que puede 
ingerirse al consumir especies acuáticas (Jackson et al., 2013; Nava-Ruíz \& Méndez-Armenta, 2011). El mercurio superó los niveles permisibles $(0,174$ $\mathrm{mg} / \mathrm{kg})$ sólo en la estación Curaray $3(0,27$ a 0,30 $\mathrm{mg} / \mathrm{kg}$ ), estando cerca al límite permisible en la estación Napo $2(0,15 \mathrm{mg} / \mathrm{kg})$ y Arabela $2(0,13$ $\mathrm{mg} / \mathrm{kg}$ ). La presencia de mercurio en los ecosistemas acuáticos amazónicos puede deberse a la explotación de oro, principalmente (Lacerda et al., 1989; Malm et al., 1990; Guyot, 1993; LIDEMA, 1993; Nriagu, 1993; GOREL-DIGESA, 2012). Ante la aparente ausencia o poca actividad mineras en el Arabela y el Curaray, la deforestación podría ser una de las principales fuentes de aporte de mercurio, liberándose de los suelos sin cobertura boscosa (claros), acabando por escorrentía en los ríos y quebradas (Roulet et al., 1999; Fostier et al., 2000). El arsénico, que también es importante por su toxicidad y cuyo origen principal en los cuerpos de agua se debe principalmente al uso de plaguicidas y conservantes de madera (Mancilla-Villa et al., 2012; Nava-Ruíz \& Méndez-Armenta, 2011) fue detectado en todas las estaciones de muestreo $(1,19-$ $4,06 \mathrm{mg} / \mathrm{kg}$ ), pero dentro de los límites permisibles. Es muy importante mencionar que la toxicidad del arsénico para los humanos depende en gran medida de la forma química en la que es ingerido (MancillaVilla et al., 2012). Los compuestos orgánicos de éste metal se encuentran en concentraciones elevadas de forma natural en los pescados, moluscos y crustáceos, son menos tóxicos que las formas inorgánicas (Nava-Ruíz \& Méndez-Armenta, 2011). En nuestro estudio, éste metal no fue registrado en las muestras de agua y peces, por lo que la concentración encontrada en el sedimento, además de estar dentro de los límites permisibles, no representa un riesgo si es que no se remueve el fondo de los ríos.

En el caso del cobre presentó concentraciones de $23,6 \mathrm{mg} / \mathrm{kg}$ y $28,7 \mathrm{mg} / \mathrm{kg}$, en las estaciones Curaray 3 y Napo 1, respectivamente. Ambos valores fueron superiores al nivel máximo recomendado por la agencia gubernamental estadounidense EPA región IV $(18.7 \mathrm{mg} / \mathrm{kg})$. El cromo fue detectado en una concentración de $38,9 \mathrm{mg} / \mathrm{kg}$ en la estación Curaray 3. Esta concentración es ligeramente superior al límite establecido por las normas ambientales canadienses $(37,3 \mathrm{mg} / \mathrm{kg})$. Así mismo, las concentraciones de níquel superaron ligeramente los niveles máximos permitidos por las normas ambientales estadounidenses $(15,9 \mathrm{mg} / \mathrm{kg})$ y canadienses $(18 \mathrm{mg} / \mathrm{kg})$ en las estaciones Curaray 2 (orilla), Curaray 3 (orilla) y Napo 2 (orilla y centro) $(18,4$ a $23,9 \mathrm{mg} / \mathrm{kg})$. Las concentraciones de metales pesados como el cromo, cadmio, plomo y zinc, siempre estuvieron dentro de los estándares permisibles internacionales (Canadá, Estados Unidos y Holanda).

\section{Aceites y grasas, HTP y metales en peces}

Los peces, y en especial las especies sedentarias, son importantes bioindicadores de la integridad de los ecosistemas acuáticos y de la exposición de la población a elementos tóxicos; por lo que nuestro estudio analizó varias especies de diferentes categorías tróficas. Tanto en creciente como en vaciante las especies colectadas, según García et al. 2008, pertenecieron a las siguientes categorías tróficas: Detritívoros (llambina Potamorhina altamazonica, yahuarachi Potamorhina latior y boquichico Prochilodus nigricans, yaraquí Semaprochilodus unitaeniatus y maparate Hypophthalmus edentatus), Omnívoros (lisa Leporinus trifasciatus, sábalo Brycon cephalus, y palometa Mylossoma duriventre y lisa Schizodon fasciatus) y Piscívoros (huapeta Hydrolycus scomberoides, tigre zúngaro Pseudoplatystoma tigrinum y doncella Pseudoplatystoma punctifer).

En los tres ríos evaluados ninguna de las especies registraron la presencia de HTP. Mientras que todas registraron la presencia de aceites y grasas (de 1213 $\mathrm{mg} / \mathrm{kg}$ en $P$. punctifer a $85376 \mathrm{mg} / \mathrm{kg}$ en $H$. edentatus), expresando el contenido natural de lípidos de estas especies. En el tejido muscular de los peces analizados no se registraron metales dañinos (cadmio, cobre, plomo, cromo y arsénico, ver Tabla 4) para la salud poblacional o ecosistémica. El mercurio sólo fue detectado en la época de vaciante, pero en concentraciones entre 7 y 16 veces por debajo de lo permisible $(0,5 \mathrm{mg} / \mathrm{kg})$. Las concentraciones registradas de $0,03-0,07 \mathrm{mg} / \mathrm{kg}$ sólo fueron reportadas para las especies $P$. tigrinum (Curaray 1), P. punctifer (Curaray 3), M. duriventre (Arabela 1) y P. nigricans (Napo 2). Las mayores concentraciones de mercurio fueron registradas en las especies migradoras de régimen piscívoro $P$. tigrinum y $P$. punctifer (a excepción de $H$. scomberoides), corroborando la tesis que refiere que este metal se bioacumula principalmente en los peces predadores (Veiga et al., 1999; Jørn 2001), porque se alimentan de sus predecesores detritívoros y omnívoros. No obstante, por ser grandes migradores (García-Dávila et al., 2013), la presencia de mercurio en los tejidos de $P$. tigrinum y $P$. punctifer puede ser circunstancial, pudiendo haberlo bioacumulado o biomagnificado en otras áreas (por ejemplo, las cabeceras del río Napo). La concentración para $P$. punctifer es cercana a lo obtenido por Beltran-Pedreros et al (2011) $(0,054$ $\mathrm{mg} / \mathrm{kg}$ ), mientras que para las demás especies del estudio obtuvieron valores que se sobreponen entre 
los niveles tróficos, lo que supuestamente se debe a la alta plasticidad trófica de las especies, una característica de los peces amazónicos, que ocurre en respuesta a la abundancia y disponibilidad de alimento resultado del ciclo hidrológico de los ríos.

Los resultados obtenidos contrastan con el estudio realizado por el GOREL-DIGESA (2012), donde se evaluó el impacto ambiental de la minería aurífera informal en los ríos Napo, Curaray y Aguarico. Dicho estudio determinó en la huapeta (Hydrolycus spp.) niveles de mercurio que alcanzaron los 3,35 mg/kg (capturados cerca de la comunidad de San Rafael, río Curaray) y $5,74 \mathrm{mg} / \mathrm{kg}$ (San Fernando, río Napo), es decir, concentraciones entre 6,7 y 11,5 veces más altas que la recomendada por la Organización Mundial de la Salud (1990). Para el mismo género Hydrolycus, el mercurio no fue detectado en nuestro estudio, pudiendo deberse a las diferentes zonas de muestreo. Esto demuestra que las diferentes concentraciones de metales registrados en las especies evaluadas muestran la influencia del factor geográfico y los patrones migratorios de los peces (Kumar et al., 2011). Otro metal registrado fue el aluminio. Si bien, tradicionalmente no es considerado un agente excesivamente tóxico, algunas investigaciones lo asocian con ciertos daños neurológicos, habiéndose incrementado progresivamente la exposición humana a este metal (en magnitud y en diversidad de fuentes), lo que introduce la incertidumbre suficiente para establecer la necesidad de evaluar el riesgo alimentario en la actualidad (Fernández et al., 2009). El aluminio fue encontrado en un rango que varió desde el nivel de la no detección en P. punctifer (Curaray 3 ) hasta los $13, .3 \mathrm{mg} / \mathrm{kg}$ observados en $P$. tigrinum (Curaray 1). El estudio realizado por el GOREL-DIGESA (2012) en el tejido muscular de los peces registró concentraciones de aluminio desde $1 \mathrm{mg} / \mathrm{kg}$ en $P$. nigricans (a la altura de la comunidad de Rango Isla) a $18,4 \mathrm{mg} / \mathrm{kg}$ en Platystomatichthys sturio. Para la huapeta (Hydrolycus spp.) reportaron concentraciones de 1,8 $\mathrm{mg} / \mathrm{kg}$ y para lisa cuatro bandas fue de $2,2 \mathrm{mg} / \mathrm{kg}$. En el presente trabajo los niveles de aluminio registrados no superan dichas concentraciones. El registro de otros metales como calcio, potasio, sodio, magnesio y manganeso pueden considerarse normales. Estos minerales son electrolitos importantes en la homeostasis interna de las células, regulando el balance iónico celular (Kumar et al., 2011).

\section{CONCLUSIONES}

En general, los resultados muestran que la calidad física y química de las agua de los ríos Curaray, Arabela y Napo (a la altura de la desembocadura del río Curaray), en relación a los parámetros evaluados durante el año 2012, muestran condiciones adecuadas para el desarrollo de la vida acuática, toda vez que las concentraciones de los metales pesados y HTP encontrados por encima de los estándares de calidad en algunas estaciones de muestreo, no comprometen la integridad de los ecosistemas acuáticos. Los elementos encontrados se concentran en los lugares de muestreo, por lo que no muestran un comportamiento de dilución río abajo desde un punto de mayor concentración. Este hecho evidencia que la presencia de los mismos está en relación al origen geológico y/o al desarrollo de algunas actividades antrópicas puntuales (no determinadas) en las zonas de muestreo, ubicadas cerca a los mayores poblados de la cuenca. Esta conclusión será corroborada con un mayor número de muestreos. 
Tabla 1. Calidad del agua registrada en siete estaciones de colecta (0: Orilla, C: Centro) localizadas en los ríos Arabela, Curaray y Napo, en época de creciente (Abril-Mayo, 2012) y vaciante (Octubre-Noviembre, 2012).

\begin{tabular}{|c|c|c|c|c|c|c|c|c|c|c|c|c|c|c|c|c|}
\hline & \multirow{2}{*}{ Parámetros Físicos y Químicos } & \multicolumn{2}{|c|}{ Curaray 1} & \multicolumn{2}{|c|}{ Curaray 2} & \multicolumn{2}{|c|}{ Curaray 3} & \multicolumn{2}{|c|}{ Arabela 1} & \multicolumn{2}{|c|}{ Arabela 2} & \multicolumn{2}{|c|}{ Napo 1} & \multicolumn{2}{|c|}{ Napo 2} & \multirow{2}{*}{$\begin{array}{c}\text { Límite Máximo } \\
\text { Permisible }\end{array}$} \\
\hline & & 0 & C & 0 & C & 0 & C & 0 & C & 0 & C & 0 & C & 0 & C & \\
\hline \multirow{12}{*}{ 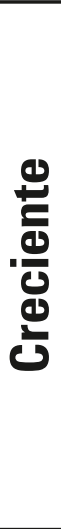 } & Transparencia (cm) & 25 & 25 & 30 & 30 & 20 & 30 & 20 & 20 & 40 & 50 & 30 & 30 & 30 & 30 & - \\
\hline & Temperatura Agua $\left({ }^{\circ} \mathrm{C}\right)$ & 24,4 & 24,4 & 25,1 & 25,1 & 26,1 & 26,1 & 24,9 & 24,9 & 25,2 & 25,2 & 25,8 & 25,7 & 25,8 & 25,7 & $\operatorname{Max} .32^{\circ} \mathrm{C}^{\mathrm{C}}$ \\
\hline & Conductividad ( $\mu \mathrm{S} / \mathrm{cm})$ & 6,5 & 6,5 & 17 & 17 & 15 & 17 & 9 & 9 & 10 & 10 & 40 & 40 & 40 & 40 & - \\
\hline & Sólidos Totales Disueltos (mg/L) & 5,5 & 5,5 & 11 & 11 & 11 & 11 & 6 & 6 & 6 & 6 & 26 & 25 & 26 & 25 & $500 \mathrm{mg} / \mathrm{L}^{\mathrm{A}}$ \\
\hline & Oxígeno Disuelto (mg/L) & 6,1 & 6,1 & 6,1 & 6,2 & 5,9 & 5,8 & 6,2 & 6,2 & 4,7 & 4,8 & 6,5 & 6,4 & 6,5 & 6,4 & $\geq 5 \mathrm{mg} / \mathrm{L}^{\mathrm{A}}$ \\
\hline & pH & 6,2 & 6,2 & 6,4 & 6,4 & 6,4 & 6,6 & 5,8 & 5,7 & 5,8 & 5,7 & 7,2 & 7,1 & 7,2 & 7,1 & $6,5-8,5^{D}$ \\
\hline & $\mathrm{CO}_{2}$ disuelto $(\mathrm{mg} / \mathrm{L})$ & 5 & 4 & 3 & 3 & 5 & 5 & 5 & 5 & 2 & 2 & 5 & 4 & 5 & 4 & - \\
\hline & Alcalinidad Total (mg/L) & 12 & 12 & 12 & 16 & 16 & 16 & 12 & 16 & 10 & 10 & 12 & 12 & 12 & 12 & - \\
\hline & Dureza Total (ppm de $\mathrm{CaCO}_{3}$ ) & 8 & 8 & 12 & 12 & 10 & 11 & 8 & 8 & 8 & 8 & 8 & 8 & 9 & 9 & - \\
\hline & Cloruros $(\mathrm{mg} / \mathrm{L})$ & 4 & 4 & 4 & 4 & 8 & 8 & 8 & 8 & 8 & 8 & 4 & 4 & 4 & 4 & $250 \mathrm{mg} / \mathrm{L}^{\mathrm{C}}$ \\
\hline & Amonio (mg/L) & 0,2 & 0,2 & $<0,2$ & $<0,2$ & 0,2 & 0,2 & 0,2 & 0.2 & 0,4 & 0.2 & 0,2 & 0.2 & 0,2 & 0,2 & $0,05 \mathrm{mg} / \mathrm{L}^{A}$ \\
\hline & Nitrito (mg/L) & $<0,05$ & $<0,05$ & $<0,05$ & $<0,05$ & $<0,05$ & $<0,05$ & $<0,05$ & $<0,05$ & $<0,05$ & $<0,05$ & $<0,05$ & $<0,05$ & $<0,05$ & $<0,05$ & $\leq 0,03 \mathrm{mg} / \mathrm{L}^{\mathrm{B}}$ \\
\hline \multirow{12}{*}{ 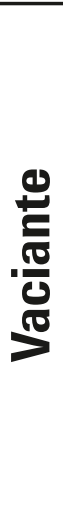 } & Transparencia $(\mathrm{cm})$ & 15 & 14 & 16 & 15 & 15 & 12 & 25 & 30 & 25 & 20 & 22 & 20 & 18 & 17 & - \\
\hline & Temperatura Agua $\left({ }^{\circ} \mathrm{C}\right)$ & 27,4 & 27,3 & 274 & 27,4 & 27,5 & 26,6 & 29,7 & 30,2 & 29,3 & 29,4 & 28,5 & 28,5 & 28,7 & 28,7 & $\operatorname{Max} .32^{\circ} \mathrm{C} C$ \\
\hline & Conductividad $(\mu \mathrm{S} / \mathrm{cm})$ & 28 & 26 & 24 & 25 & 20 & 22 & 21 & 21 & 19 & 18 & 56 & 56 & 23 & 56 & - \\
\hline & Sólidos Totales Disueltos (mg/L) & 18 & 17 & 16 & 16 & 13 & 14 & 14 & 14 & 11 & 12 & 37 & 36 & 15 & 36 & $500 \mathrm{mg} / \mathrm{L}^{\mathrm{A}}$ \\
\hline & Oxígeno Disuelto (mg/L) & 6,6 & 6,5 & 6,4 & 6,2 & 6,4 & 6 & 6 & 6,3 & 5,7 & 5,8 & 6,6 & 6,4 & 6,4 & 6,5 & $\geq 5 \mathrm{mg} / \mathrm{e}$ \\
\hline & $\mathrm{pH}$ & 7,0 & 7,1 & 7,1 & 7,1 & 7,4 & 7,5 & 6,9 & 6,9 & 6,9 & 7 & 7,5 & 7,6 & 7,3 & 7,6 & $6,5-8,5^{D}$ \\
\hline & $\mathrm{CO}_{2}$ disuelto $(\mathrm{mg} / \mathrm{L})$ & 5 & 5 & 4 & 5 & 5 & 4 & 7 & 5 & 5 & 5 & 3 & 2 & 4 & 5 & - \\
\hline & Alcalinidad Total (mg/L) & 16 & 16 & 16 & 16 & 12 & 12 & 8 & 12 & 12 & 12 & 24 & 24 & 16 & 24 & - \\
\hline & Dureza Total (ppm de $\mathrm{CaCO}_{3}$ ) & 12 & 12 & 12 & 12 & 8 & 8 & 8 & 8 & 8 & 8 & 20 & 20 & 20 & 20 & - \\
\hline & Cloruros (mg/L) & 8 & 8 & 8 & 8 & 8 & 8 & 4 & 8 & 8 & 8 & 8 & 8 & 8 & 8 & $250 \mathrm{mg} / \mathrm{L}^{\mathrm{C}}$ \\
\hline & Amonio (mg/L) & 0,2 & 0,2 & 0,4 & 0,4 & $<0,2$ & $<0,2$ & $<0,2$ & $<0,2$ & $<0,2$ & $<0,2$ & $<0,2$ & $<0,2$ & $<0,2$ & $<0,2$ & $0,05 \mathrm{mg} / \mathrm{L}^{\mathrm{A}, \mathrm{B}}$ \\
\hline & Nitrito (mg/L) & $<0,05$ & $<0,05$ & $<0,05$ & $<0,05$ & $<0,05$ & $<0,05$ & $<0,05$ & $<0,05$ & $<0,05$ & $<0,05$ & $<0,05$ & $<0,05$ & $<0,05$ & $<0,05$ & $\leq 0,03 \mathrm{mg} / \mathrm{L}^{\mathrm{B}}$ \\
\hline
\end{tabular}

Leyenda: A, B, C y D son los códigos asignados de los estándares de calidad ambiental mencionados en la sección "Análisis de las muestras e interpretación de los resultados". 
Tabla 2. Concentración de metales (mg/L) en aguas superficiales colectadas en siete estaciones de muestreo (0: Orilla, C: Centro) localizadas en los ríos Arabela, Curaray y Napo, en época de creciente (Abril-Mayo, 2012) y vaciante (Octubre-Noviembre, 2012).

\begin{tabular}{|c|c|c|c|c|c|c|c|c|c|c|c|c|c|c|c|c|}
\hline & \multirow[t]{2}{*}{ Metales } & \multicolumn{2}{|c|}{ Curaray 1} & \multicolumn{2}{|c|}{ Curaray 2} & \multicolumn{2}{|c|}{ Curaray 3} & \multicolumn{2}{|c|}{ Arabela 1} & \multicolumn{2}{|c|}{ Arabela 2} & \multicolumn{2}{|c|}{ Napo 1} & \multicolumn{2}{|c|}{ Napo 2} & \multirow{2}{*}{$\begin{array}{l}\text { Límite Máximo } \\
\text { Permisible (mg/L) }\end{array}$} \\
\hline & & 0 & C & 0 & C & 0 & C & 0 & C & 0 & C & 0 & C & 0 & C & \\
\hline \multirow{12}{*}{ ڤִ } & Aluminio & 0,89 & 0,90 & 0,68 & 1,14 & 0,49 & 0,81 & 0,22 & 0,35 & 0,38 & 0,39 & 0,32 & 1,06 & 0,80 & 0,51 & $0,1^{C} ; 0,2^{D}$ \\
\hline & Bario & 0,057 & 0,046 & 0,039 & 0,047 & 0,038 & 0,035 & 0,015 & 0,018 & 0,022 & 0,018 & 0,02 & 0,033 & 0,041 & 0,025 & $1^{A}$ \\
\hline & Calcio & 2,676 & 2,589 & 2,082 & 2,2 & 1,938 & 2,094 & 1,976 & 1,207 & 1,331 & 1,102 & 4,121 & 6,015 & 1,836 & 4,332 & - \\
\hline & Cobre & N.D & 0,011 & N.D & $\mathrm{N}, \mathrm{D}$ & N.D & 0,011 & N.D & N.D & N.D & N.D & 0,051 & N.D & 0,051 & N.D & $0,02^{A} ; 2,0^{D}$ \\
\hline & Hierro & 2,02 & 1,87 & 1,62 & 2,09 & 1,36 & 1,79 & 0,90 & 1,28 & 1,70 & 1,68 & 0,66 & 1,58 & 1,91 & 0,96 & $0,3^{D}$ \\
\hline & Potasio & 0,53 & 0,51 & 0,47 & 0,56 & 0,44 & 0,41 & 0,33 & 0,15 & 0,36 & 0,21 & 0,56 & 1,12 & 0,52 & 0,62 & - \\
\hline & Magnesio & 1,029 & 0,874 & 0,775 & 0,855 & 0,737 & 0,766 & 0,454 & 0,344 & 0,416 & 0,403 & 0,85 & 1,424 & 0,757 & 0,963 & - \\
\hline & Manganeso & 0,13 & 0,08 & 0,08 & 0,08 & 0,067 & 0,06 & 0,07 & 0,05 & 0,08 & 0,08 & 0,03 & 0,05 & 0,10 & 0,05 & $0,1^{C, D}$ \\
\hline & Sodio & 1,37 & 1,22 & 1,05 & 1,21 & 1,08 & 1,1 & 0,87 & 0,69 & 0,69 & 0,62 & 1,26 & 2,03 & 0,91 & 1,37 & - \\
\hline & Fósforo & 0,05 & 0,05 & 0,04 & 0,05 & 0,03 & 0,06 & 0,03 & 0,02 & 0,03 & 0,03 & 0,05 & 0,09 & 0,04 & 0,07 & $0,1^{D}$ \\
\hline & Vanadio & 0,005 & 0,005 & 0,004 & 0,005 & 0,003 & 0,004 & 0,002 & 0,002 & 0,002 & 0,002 & 0,002 & 0,005 & 0,005 & 0,003 & $0,1^{\mathrm{C}}$ \\
\hline & Zinc & 0,024 & 0,042 & 0,035 & 0,027 & 0,023 & 0,033 & 0,042 & 0,029 & 0,026 & 0,014 & 0,057 & 0,018 & 0,022 & 0,04 & $0,3^{\mathrm{A}}$ \\
\hline \multirow{16}{*}{ 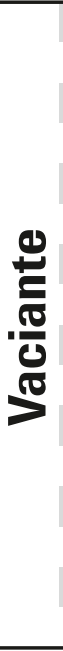 } & Aluminio & 1,97 & 1,8 & 1,96 & 1,87 & 0,98 & 1,13 & 0,15 & 0,18 & 0,22 & 0,25 & 1,66 & 1,25 & 0,95 & 1,30 & $0,1^{C} ; 0,2^{D}$ \\
\hline & Bario & 0,08 & 0,08 & 0,07 & 0,08 & 0,04 & 0,05 & 0,02 & 0,02 & 0,02 & 0,02 & 0,05 & 0,05 & 0,04 & 0,05 & $1^{A}$ \\
\hline & Calcio & 3,25 & 3,86 & 2,75 & 3,14 & 2,06 & 2,41 & 1,73 & 1,88 & 1,56 & 1,83 & 7,16 & 7,44 & 2,55 & 5,53 & - \\
\hline & Cobalto & 0,003 & 0,003 & 0,002 & 0,002 & $N, D$ & $\mathrm{~N}, \mathrm{D}$ & $\mathrm{N}, \mathrm{D}$ & N.D & N.D & N.D & 0,001 & 0,001 & N.D & N.D & $0,02^{C}$ \\
\hline & Cromo & 0.004 & 0,004 & 0,004 & 0,004 & N.D & N.D & N.D & N.D & N.D & N.D & N.D & N.D & N.D & N.D & $0,05^{A}$ \\
\hline & Cobre & 0,006 & 0,006 & 0,006 & 0,006 & 0,003 & 0,004 & N.D & N.D & N.D & N.D & 0,007 & 0,006 & 0,003 & 0,006 & $0,02^{A} ; 2,0^{D}$ \\
\hline & Hierro & 3,52 & 3,27 & 3,51 & 3,36 & 1,76 & 1,96 & 1,66 & 1,56 & 1,47 & 1,53 & 2,17 & 1,67 & 1,59 & 2,01 & $0,3^{D}$ \\
\hline & Potasio & 0,94 & 0,88 & 0,87 & 0,89 & 0,78 & 0,77 & 0,51 & 0,51 & 0,45 & 0,47 & 1,47 & 1,44 & 0,82 & 1,25 & - \\
\hline & Magnesio & 1,42 & 1,40 & 1,18 & 1,25 & 0,83 & 0,91 & 0,63 & 0,62 & 0,53 & 0,54 & 1,62 & 1,52 & 0,88 & 1,34 & - \\
\hline & Manganeso & 0,16 & 0,15 & 0,15 & 0,15 & 0,08 & 0,09 & 0,07 & 0,07 & 0,07 & 0,06 & 0,10 & 0,09 & 0,06 & 0,10 & $0,1^{C, D}$ \\
\hline & Sodio & 1,51 & 3,7 & 1,26 & 1,33 & 1,05 & 1,1 & 1,29 & 1,23 & 0,98 & 1,07 & 2,01 & 2,0 & 1,12 & 1,65 & - \\
\hline & Níquel & 0,002 & 0,002 & 0,002 & 0,002 & N.D & N.D & N.D & N.D & N.D & N.D & N.D & N.D & N.D & N.D & $0,025^{A}$ \\
\hline & Fósforo & 0,09 & 0,09 & 0,08 & 0,08 & 0,05 & 0,05 & 0,03 & 0,02 & 0,02 & 0,03 & 0,16 & 0,13 & 0,05 & 0,12 & $0,1^{D}$ \\
\hline & Antimonio & $N, D$ & N.D & N.D & N.D & 0.017 & N.D & N.D & N.D & N.D & N.D & N.D & N.D & N.D & N.D & $0,006^{D}$ \\
\hline & Vanadio & 0,011 & 0,01 & 0,01 & 0,009 & 0,005 & 0,006 & ND & ND & 0,001 & 0,001 & 0,007 & 0,006 & 0,005 & 0,006 & $0,1^{c}$ \\
\hline & Zinc & 0,027 & 0,025 & 0,017 & 0,033 & 0,013 & 0,016 & 0,018 & 0,013 & 0,01 & 0,02 & 0,015 & 0,036 & 0,018 & 0,018 & $0,3^{\mathrm{A}}$ \\
\hline
\end{tabular}

Leyenda: A, C y D son los códigos asignados de los estándares de calidad ambiental mencionados en la sección "Análisis de las muestras e interpretación de los resultados". N.D.: No detectado 
Tabla 3. Concentraciones de metales (mg/kg) en muestras de sedimentos colectados en siete estaciones de trabajo (0: Orilla, C: Centro) localizados en los ríos Arabela, Curaray y Napo, en época de creciente (Abril-Mayo) y vaciante (Octubre-Noviembre).

\begin{tabular}{|c|c|c|c|c|c|c|c|c|c|c|c|c|c|c|c|c|}
\hline \multicolumn{2}{|c|}{ Metales } & \multicolumn{2}{|c|}{ Curaray 1} & \multicolumn{2}{|c|}{ Curaray 2} & \multicolumn{2}{|c|}{ Curaray 3} & \multicolumn{2}{|c|}{ Arabela 1} & \multicolumn{2}{|c|}{ Arabela 2} & \multicolumn{2}{|c|}{ Napo 1} & \multicolumn{2}{|c|}{ Napo 2} & \multirow{2}{*}{$\begin{array}{l}\text { Límite Máximo } \\
\text { Permisible } \\
\text { (mg/kg) }\end{array}$} \\
\hline & & 0 & C & 0 & C & 0 & C & 0 & C & 0 & C & 0 & C & 0 & C & \\
\hline \multirow{15}{*}{ 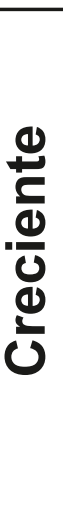 } & Aluminio & 2505 & 1930 & 7031 & 1438 & 23018 & 68432 & 2588 & 2325 & 651,3 & 2662 & 14241 & 4045 & 11476,4 & 3498 & - \\
\hline & Bario & 43,9 & 41,2 & 100 & 28,8 & 34,9 & 110 & 31,8 & 329 & 9 & 28,8 & 131 & 51,2 & 30,9 & 48,4 & $200^{G}$ \\
\hline & Calcio & 765 & 617 & 1183 & 416 & 473 & 1593 & 718 & 727 & 250 & 547 & 4149 & 2626 & 460 & 2506 & - \\
\hline & Cobalto & 5,5 & 7,3 & 9,2 & 5,6 & 6,7 & 9,3 & 4,4 & 4,1 & 1,3 & 4,9 & 10,9 & 7,9 & 1,2 & 7,6 & $20^{G}$ \\
\hline & Cromo & 14,6 & 7,1 & 18,6 & 4,6 & 7,3 & 21,3 & 5,5 & 5,7 & 2,5 & 6,6 & 17,5 & 10,7 & 15,9 & 9,2 & $37,3^{E}$ \\
\hline & Cobre & 6,4 & 4,3 & 14,6 & 2,9 & 3,6 & 15,7 & 7,8 & 7,8 & 2,6 & 6,8 & 39,3 & 7,6 & 9,9 & 6,9 & $35,7^{\mathrm{E}}$ \\
\hline & Hierro & 8455 & 6688 & 18,22 & 5212 & 7053 & 15581 & 8608 & 7638 & 3088 & 10908 & 24706 & 17290 & 14017 & 16478 & - \\
\hline & Potasio & 104 & 85,1 & 171 & 84,1 & 101 & 220 & 154 & 119 & 58,8 & 138 & 1128 & 367 & 290 & 329 & - \\
\hline & Magnesio & 1184 & 879 & 1526 & 571 & 771 & 1947 & 628 & 552 & 173 & 454 & 5284 & 2544 & 401 & 2457 & - \\
\hline & Manganeso & 158 & 274 & 367 & 207 & 246 & 3777 & 219 & 202 & 55,1 & 206 & 533 & 262 & 18,3 & 250 & - \\
\hline & Sodio & N.D & N.D & N.D & N.D & N.D & N.D & N.D & N.D & N.D & N.D & 229 & 171 & 74 & 96.1 & - \\
\hline & Níquel & 8,2 & 8,2 & 11,4 & 5,4 & 6,9 & 15,1 & 5 & 4,6 & 2,5 & 3,9 & 17 & 10,5 & 3,1 & 7,2 & $18^{\mathrm{E}}$ \\
\hline & Fósforo & 104 & 89,4 & 215 & 60,4 & 79,2 & 216 & 183 & 156 & 64,4 & 226 & 857 & 726 & 54,5 & 767 & - \\
\hline & Plomo & $\mathrm{N}, \mathrm{D}$ & N.D & 9.4 & N.D & N.D & 7.5 & 5.3 & 4,3 & $\mathrm{~N}, \mathrm{D}$ & 6,1 & 12,3 & 4 & 13,2 & 4 & $35^{\mathrm{E}}$ \\
\hline & Zinc & 35,3 & 35 & 50,9 & 27,9 & 30 & 63,1 & 3,8 & 32,8 & 15,6 & 27,4 & 86,6 & 46,1 & 36,8 & 44,8 & $123^{\mathrm{E}}$ \\
\hline \multirow{18}{*}{$\underset{v}{\frac{0}{0}}$} & Aluminio & 9471 & 5268 & 20674 & 4540 & 23908 & 2231 & 3362 & 9830 & 2219 & 12023 & 13444 & 6917 & 20550 & 2585 & - \\
\hline & Arsénico & 1,51 & 1,57 & 3,7 & 1,91 & 3,83 & 1,29 & 1,44 & 4,06 & 1,63 & 2,88 & 3,58 & 1,19 & 2,31 & 1,96 & $5,9^{\mathrm{E}}$ \\
\hline & Bario & 62 & 36,0 & 130 & 36,1 & 168 & 19,8 & 18,6 & 36,7 & 27,8 & 66,9 & 102 & 59,5 & 116 & 58,0 & $200^{G}$ \\
\hline & Calcio & 1277 & 798 & 217 & 583 & 192 & 372 & 428 & 593 & 561 & 2062 & 4506 & 3299 & 1890 & 5027 & - \\
\hline & Cobalto & 9,2 & 8,1 & 13,4 & 8,4 & 20,0 & 4,7 & 2,7 & 10,7 & 3,7 & 8,7 & 122 & 10,2 & 123 & 20,9 & $20^{G}$ \\
\hline & Cromo & 21,0 & 10,2 & 32,2 & 8,9 & 38,9 & 5,0 & 4,6 & 128 & 6,7 & 13,8 & 16,8 & 9,8 & 30,7 & 31,8 & $37,3^{E}$ \\
\hline & Cobre & 7,2 & 3,2 & 19,6 & 3,2 & 23,6 & 2,2 & 2,3 & 6,8 & 4,6 & 9,9 & 28,7 & 7,8 & 17,3 & 9,8 & $35,7^{\mathrm{E}}$ \\
\hline & Hierro & 4328 & 9002 & 26634 & 8424 & 32327 & 4704 & 4776 & 20584 & 7572 & 18643 & 26584 & 20368 & 26083 & 48752 & - \\
\hline & Potasio & 323 & 176 & 880 & 143 & 921 & 70,3 & 147 & 561 & 296 & 837 & 1330 & 512 & 711 & 693 & - \\
\hline & Magnesio & 1512 & 869 & 2263 & 762 & 2576 & 433 & 350 & 743 & 595 & 1212 & 7022 & 2194 & 2221 & 2837 & - \\
\hline & Manganeso & 284 & 289 & 637 & 301 & 776 & 197 & 120 & 502 & 150 & 427 & 434 & 309 & 418 & 474 & - \\
\hline & Molibdeno & 0,26 & 0,2 & 0,72 & 0,22 & 0,85 & 0,18 & 0,2 & 0,66 & 0,3 & 0,56 & 0,68 & 0,22 & 0,52 & 0,24 & $10^{G}$ \\
\hline & Sodio & 51,9 & 40,7 & 92 & 25,5 & 721 & 14 & 23,2 & 28,4 & 29,6 & 47,4 & 400 & 298 & 123 & 543 & - \\
\hline & Níquel & 122 & 8,1 & 20,1 & 7,7 & 23,9 & 4,5 & 2,4 & 5,1 & 3,8 & 8,2 & 15,0 & 10,8 & 18,4 & 18,4 & $18^{\mathrm{E}}$ \\
\hline & Fósforo & 136 & 86,2 & 344 & 825 & 387 & 52,8 & 79,5 & 301 & 126 & 284 & 899 & 847 & 318 & 1065 & - \\
\hline & Plomo & 6,3 & 3,9 & 14,8 & 3,8 & 18,6 & 2,4 & 4,3 & 10,8 & 6,3 & 12,8 & 10,8 & 5,4 & 14,5 & 6,2 & $35^{\mathrm{E}}$ \\
\hline & Zinc & 40,6 & 29,4 & 84,2 & 32,3 & 98,9 & 24,2 & 18,7 & 35 & 27,6 & 53,6 & 71 & 43,6 & 74,9 & 72,4 & $123^{\mathrm{E}}$ \\
\hline & Mercurio & 0,06 & 0,04 & 0,09 & 0,07 & 0,27 & 0,30 & 0,12 & 0,12 & 0,13 & 0,13 & 0,10 & 0,11 & 0,15 & 0,13 & $0,174^{\mathrm{E}}$ \\
\hline
\end{tabular}

Leyenda: E y G son los códigos asignados de los estándares de calidad ambiental mencionados en la sección “Análisis de las muestras e interpretación de los resultados".

N.D.: No detectado 
Tabla 4. Concentraciones de metales pesados $(\mathrm{mg} / \mathrm{kg})$ presentes en tejidos de peces capturados en siete estaciones de trabajo ubicados en los ríos Arabela, Curaray y S. Napo, en época de creciente (Abril-Mayo) y vaciante (Octubre-Noviembre). Fuente: ENVIROLAB PERU S.A.C. Método de determinación de metales: EPA6010B. Método de determinación de mercurio: CVAFS basado en EPA 1631.

\begin{tabular}{lcccccccc}
\hline METALES & Curaray 1 & Curaray 2 & Curaray 3 & Arabela 1 & Arabela 2 & Napo 1 & Napo 2 & $\begin{array}{c}\text { Límite Máx. } \\
\text { Permisible }\end{array}$ \\
\hline \multicolumn{1}{c}{ CRECIENTE } & P. altamazonica & H. scomberoides & L. trifasciatus & P. latior & B. cephalus & P. nigricans & M. duriventre & \\
\hline Cromo & N.D & N.D & 0.6 & N.D & N.D & N.D & N.D & $1,1 \mathrm{mg} / \mathrm{kg}^{\mathrm{L}}$ \\
Hierro & 12,0 & N.D & 12,3 & 7,1 & N.D & 21,0 & 8,3 & $?$ \\
Manganeso & N.D & 0,1 & N.D & N.D & N.D & N.D & N.D & $?$ \\
Zinc & 11,0 & 7,1 & N.D & 13,0 & 8,2 & 10,2 & 14,0 & $50 \mathrm{mg} / \mathrm{kg}{ }^{\prime}$ \\
\hline
\end{tabular}

\begin{tabular}{|c|c|c|c|c|c|c|c|c|}
\hline VACIANTE & P. tigrinum & S. fasciatus & P. punctifer & M. duriventre & S. unitaeniatus & H. edentatus & P. nigricans & \\
\hline Aluminio & 13,3 & 4.1 & N.D & 4,6 & 2,0 & 3,4 & 4,3 & $?$ \\
\hline Bario & N.D & 0,7 & N.D & N.D & N.D & N.D & N.D & $?$ \\
\hline Calcio & 146,9 & 164,5 & 166,2 & 590,2 & 327,7 & 79,5 & 462,0 & $?$ \\
\hline Hierro & 31,5 & N.D & N.D & 8,5 & N.D & N.D & 7,7 & $?$ \\
\hline Potasio & 3133,8 & 1602,6 & 2648,9 & 1655,1 & 1230 & 1133,2 & 1480 & $?$ \\
\hline Magnesio & 252,7 & 178,3 & 227,7 & 205,3 & 159,4 & 153,8 & 168,5 & $?$ \\
\hline Manganeso & 0,5 & 0,8 & 0,3 & 0,9 & 0,7 & 0,3 & 0,5 & $?$ \\
\hline Sodio & 275,5 & 155,3 & 292,3 & 225,5 & 151,6 & 116,1 & 134,5 & $?$ \\
\hline Fósforo & 1606,1 & 917,5 & 1409,2 & 1191,8 & 800,3 & 705,8 & 993,1 & $?$ \\
\hline Silicio & 14,7 & 10,3 & $\mathrm{~N}, \mathrm{D}$ & 9,5 & N.D & N.D & 6,9 & $?$ \\
\hline Estroncio & 0,8 & 0,6 & 0,5 & 2,0 & 1,4 & 0,2 & 0,8 & $?$ \\
\hline Titanio & 1,5 & N.D & N.D & N.D & N.D & N.D & N.D & $?$ \\
\hline Zinc & 6,4 & 4,2 & 5,6 & 5,8 & 4,6 & 4,4 & 6,1 & $50 \mathrm{mg} / \mathrm{kg}^{\prime}$ \\
\hline Mercurio & 0,06 & N.D & 0,07 & 0,03 & N.D & N.D & 0,04 & $0,5 \mathrm{mg} / \mathrm{kg}^{\mathrm{J}}$ \\
\hline
\end{tabular}

Leyenda: I, J y L son los códigos asignados de los estándares de calidad ambiental mencionados en la sección

"Análisis de las muestras e interpretación de los resultados". N.D.: No detectado 


\section{REFERENCIA BIBLIOGRÁFICAS}

ANA (Autoridad Nacional del Agua). 2012. Informe Técnico $\mathrm{N}^{\circ}$ 018-2012-ANA-DGCRH/CGEL: Monitoreo participativo e identificación de fuentes contaminantes de la calidad de agua superficial de la cuenca del Nanay. 69pp.

Beltran-Pedreros, S.; Zuanon, J.; Rosseval Leite, R.; Pacheco, J. R.; Barros, A.; Forsberg, B. 2011. Mercury bioaccumulation in fish of commercial importance from different trophic categories in an Amazon floodplain lake. Neotropical Ichthyology, 9(4): 901-908.

Christophoridis, C.; Dedepsidis, D.; Fytianos, K. 2009. Occurrence and distribution of selected heavy metals in the surface sediments of Thermaikos Gulf, N. Greece.Assessment using pollution indicators. Journal of Hazardous Materials, 168: 1082-1091.

CONAMA (Conselho Nacional de Meio Ambiente do Brasil). Disponible en la página web: http://www.mma.gov.br/conama. Acceso: 03/03/2013.

Diagomanolin, V.; Farhang, M.; Ghazi-Khansari, M.; Jafarzadeh, N. 2004. Heavy metals (Ni, Cr, $\mathrm{Cu})$ in the Karoon waterway river, Iran. Toxicology Letter, 151: 63-68.

Driscoll, C.; Mason, R.; Man Chan, H.; Jacob,D. y Nicola Pirrone. 2013. Mercury as a Global Pollutant: Sources, Pathways, and Effects. . Environmental Science \& Technology, 47(10): 4967-4983.

EPA (Environmental Protection Agency). 1995. Ecological Screening Values - ESV. In: Supplemental Guidance to RAGS: Region 4 Bulletins-Ecological Risk Assessment, Bulletin No.2. Atlanta, Georgia. November 1995. http://www.epa.gov/region4/wastepgs/ofiecser/ otsguid.html. Acceso: 07/03/2013.

EPA (Environmental Protection Agency). 1992. Test methods for evaluating solid waste, physical/chemical methods. USEPA, SW-846, 3rd ed. United States Environmental Protection Agency.

Ferreira, E.; Zuanon, J.; Forsberg B.; Goulding, M.; Briglia-Ferreira, S. 2007. Río branco, peixes, ecología e conservaçao de Roraima. Amazon Conservation Association (ACA), Instituto Nacional de Pesquisas da Amazônia (INPA), Sociedad Civil Mamirauá. 201pp.

Fostier, A.H.; Forti, M.C.; Guimaraes, J.R.D.; Melfi, A.J.; Boulet, R.; Espirito Santo, C.M.; Krug, J.F. 2000. Mercury fluxes in a natural forested Amazonian catchment (Serra do Navio, Amapá
State, Brazil). Science of the Total Environment, 260(1-3): 201-211.

García, A.; Tello, S.; Vargas, G.; Duponchelle,F. Patterns of commercial fish landings in the Loreto region (Peruvian Amazon) between 1984 and 2009. Fish Physiology and Biochemistry, 35(1): 53-67.

García-Dávila, C.; Duponchelle, F.; Castro-Ruiz, D.; Villacorta, J.; Quérouil, S.; ChotaMacuyama, W.; Núñez, J.; Römer, U.; CarvajalVallejos, F.; Renno, J-F. 2013. Molecular identification of a cryptic species in the Amazonian predatory catfish genus Pseudoplatystoma (Bleeker, 1962) from Peru. Genetica, 141(7-9): 347-58.

GOREL-DIGESA (Gobierno Regional de Loreto Dirección General de Salud Ambiental). 2012. Impacto socio ambiental de la minería aluvial en la región Loreto. Documento Técnico. Dirección Regional de Energía y Minas de Loreto. Gobierno Regional de Loreto. Iquitos, Perú. 103 pp.

Granado, C. 2000. Ecología de comunidades, el paradigma de los peces de agua dulce. Universidad de Sevilla. España. 282 pp.

Guyot, J.L. 1993. Hydrogeochemistry of the Bolivian Amazon Rivers. Ph.D. Thesis ORSTOM France. 261pp.

Jackson, T.; Telmer, K.; Muir, D. 2013. Massdependent and mass-independent variations in the isotope composition of mercury in cores from lakes polluted by a smelter: Effects of smelter emissions, natural processes, and their interactions. Chemical Geology, 352: 27-46.

Jørn, M.M. 2001. Assessing mercury contamination in the Amazon Basin. Technical Report. 26pp.

Kumar, B.; Mukherjee, B.D.; Kumar, S.; Mishra, M.; Prakash, D.; Singh, S.K.; Sharma, C.S. 2011. Bioaccumulation of heavy metals in muscle tissue of fishes from selected aquaculture ponds in east Kolkata wetlands. Annals of Biological Research, 2(5): 125-134.

Lacerda, L.D.; Pfeiffer, W.C.; Ott, A.T.; Silveira, E.G. 1989. Mercury contamination in the Madeira river, Amazon-Hg inputs to the environment. Biotropica, 21:91-93.

LIDEMA (Liga de Defensa del Medio Ambiente). 1993. Estudio de impacto ambiental por la explotación de oro en la región de Nueva Esperanza, Araras, del departamento de Pando. LIDEMA(eds.). La Paz, Bolivia. 175pp.

MacDonald, D.D.; Ingersoll, C.G.; Berger, T.A. 2000. Development and evaluation of consensus-based sediment quality guidelines for 
freshwater ecosystems. Archives of environmental contamination and toxicology, 39: 20-31.

Maco, J. 2006. Tipos de ambientes acuáticos de la Amazonía peruana. Folia amazónica, 15 (1-2): 131-140.

Malm, O.; Pfeiffer, W.C.; Souza, C.M.M.; Reuther, R. 1990. Mercury pollution due to gold mining in the Madeira river basin, Brazil. Ambio, 19(1): 1115.

Mancilla-Villa, O.; Ortega-Escobar, H.; RamírezAyala, C.; Uscanga-Mortera, E.; Ramos-Bello, R. y Reyes-Ortigoza, A. 2012. Metales pesados totales y arsénico en el agua para riego de Puebla y Veracruz, México. Revista Internacional de Contaminación Ambiental, 28(1): 39-48.

MHSPE (Ministry of Housing, Spatial Planning, and Environment). 1994. Intervention values and Target values - Soil quality standards. Directorate General for Environmental Protection.Department of Soil Protection. The Hague, The Netherlands. 98pp.

Ministério da Saúde do Brasil. 1965. Decreto no 55.871, de 26 de março de 1965. In: Legislação federal do setor da saúde. 2da Ed. Brasília, Consultoria Jurídica, 1977; 2: 498-545.

MINAM (Ministerio del Ambiente). 2008a. Decreto Supremo $\mathrm{N}^{\circ}$ 002-2008. Estándares de Calidad Ambiental (ECA) para Aguas de Categoría 1: Poblacional y Recreacional. A1: Aguas que pueden ser potabilizadas con desinfección. Ministerio del Ambiente del Perú.

MINAM (Ministerio del Ambiente). 2008b. Decreto Supremo $\mathrm{N}^{\circ}$ 002-2008. Estándares de Calidad Ambiental (ECA) para Aguas de Categoría 4: Conservación del Ambiente Acuático. Ministerio del Ambiente del Perú.

Nava-Ruíz, C.; Méndez-Armenta, M. 2011. Efectos neurotóxicos de metales pesados (cadmio, plomo, arsénico y talio). Archivos de Neurociencias (Mex), 16(3): 140-147.

Nriagu, J.O. 1993. Mercury pollution from silver mining in colonial South America. In: Proceedings. Perspectives far Environmental Geochemistry in Tropical Countries. Niteroi, Brazil. Abrão, J.J., Wasserman, J.C. and SilvaFilho, E.V. (eds.), 365-368pp.

Organización Mundial de la Salud (OMS). 1990. Methylmercury: Environmental Health Criteria, Vol. 101. World Health Organization, Geneva.

Organización Mundial de la Salud (OMS). 2006. Guías para la calidad del agua potable. Primer apéndice a la Tercera edición. Volumen 1. Ginebra, Suiza. 408pp. 
